\title{
One-step Reversal Technique towards Hydrophilic Surface Modification of Polydimethylsiloxane-co-Polyurethane (PDMS-PU)
}

ISSN: 2576-8840

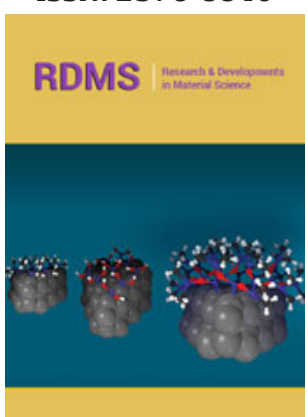

*Corresponding author: Mulenga Kalulu, School of Chemistry and Chemical Engineering, Southeast University, Jiangsu Province Hi-Tech Key Laboratory for Biomedical Research Jiangning, P R China

Submission: 眥June 16, 2020

Published: 眥July 07, 2020

Volume 13 - Issue 4

How to cite this article: Mulenga Kalulu, Richard Ngulube. One-step Reversal Technique towards Hydrophilic Surface Modification of Polydimethylsiloxaneco-Polyurethane (PDMS-PU). Res Dev Material Sci. 13(4). RDMS.000820. 2020. DOI: 10.31031/RDMS.2020.13.000820

Copyright@ Mulenga Kalulu. This article is distributed under the terms of the Creative Commons Attribution 4.0 International License, which permits unrestricted use and redistribution provided that the original author and source are credited.

\author{
Mulenga Kalulu ${ }^{1,2,3 *}$ and Richard Ngulube ${ }^{1,2}$
}

${ }^{1}$ School of Chemistry and Chemical Engineering, Southeast University, P R China

${ }^{2}$ Department of Chemistry, University of Zambia, Zambia

${ }^{3}$ Institute of Basic and Biomedical Sciences School, Levy Mwanawasa Medical University, Zambia

\begin{abstract}
Polydimethylsiloxane (PDMS) materials' surface is naturally hydrophobic due to their hydrophobic siloxane structure. Modifying the hydrophobic surface of PDMS materials to hydrophilic is a key step for its application in biomaterial and biomedical applications. In this work, a novel, simple, environment friendly and cost effective method for hydrophilic surface modification via one-step hydrolysis of hydrophobic Si-O bond to generate hydrophilic hydroxyl group was proposed. PDMS-PU films was prepared by ultraviolet copolymerization of PDMS-HEMA macromere and the hydrophilizing reagent 2(trimethylsiloxy) ethyl methacrylate (TMSEMA). The as-prepared PDMS-PU film surface was subjected to reversal treatment using potassium hydroxide $(\mathrm{KOH})$ solution to generate hydroxyl groups on the surface. The water contact angle (WCA), ATR-FTIR, equilibrium water content (EWC), SEM, mechanical properties and protein adsorption (PA) analysis were conducted on PDMS-PU films. The TMSEMA content had no obvious effect on the WCA, EWC, surface morphology and PA value of the pristine PDMS-PU film, but increased the mechanical strength and elongation at break. After subjection to the reversal treatment, the WCA deceased from $108^{\circ}$ to $16^{\circ}$ while the EWC value increased to $13 \%$ when the content of TMSEMA was $16 \%$. The IR spectrum proved reverse reaction generated the carboxylate and hydroxyl groups on the surface. The hydrophilicity was stable over a period of study (days) while the defects on the surface of the film were observed after treatment. The protein adsorption, tensile strength and elongation at break were also investigated.
\end{abstract}

Keywords: Polydimethylsiloxane, 2-(trimethylsiloxy) ethyl methacrylate; Reversal technique; Hydrophilic; Protein adsorption

\section{Introduction}

Polydimethylsiloxane (PDMS) has in the last few decades been considered as the most prevalent molding materials for the fabrication of various micro devices for biological and biomedical research and applications, due to the prominent features of non-toxicity, low cost, chemical inertness, gas permeability, optical transparency, functionalized surface and mold ability [1-4]. Despite the above appealing properties, PDMS is naturally hydrophobic and this confines its wide use in several applications including biocompatible devices, due to its tendency to absorb some hydrophobic molecules, cells and proteins onto its surface. Therefore, there is pressing need to improve the hydrophilicity of PDMS materials' surface.

In response to this, many researchers have attempted to modify the hydrophobic surface of PDMS materials to hydrophilic with oxygen-based plasma treatment being the most simple and commonly used technique [5-8]. However, the PDMS surface treated by oxygen-based plasma recovers its hydrophobicity within few hours due to the migration of the PDMS oligomers from the bulk to the air-surface interface. In order to reduce the rate of hydrophobic recovery of PDMS modified by oxygen-based plasma, water has been applied as a storing 
solution, but this method of storage is not suitable if PDMS is to be used to fabricate micro fluidic devices [5]. Therefore, there is need to find simple and cost effective methods for increasing and preserving the hydrophilicity to the maximum possible extent for a longer period. Various methods such as sol-gel coating [9], thermal aging [10], deliberate protein adsorption [11], salinization [12], radiation-induced polymerization [13], layer-by-layer (LBL) deposition $[14,15]$ and many others have been proposed for achieving long-term hydrophilic surface modification. These methods are tedious, time-consuming including requiring of well-trained technicians to execute them.

Recently, Tian et al. [8] studied the hydrophobic and hydrogel's adhesion through hydrophilic modification and interfacial segregation by utilizing a combination of plasma treatments and rheological modifier. They showed that, the adhesion between oxygen plasma-treated PDMS and the hydrogel increased with time and reached a stable value after six days and the WCA remained constant during that time, suggesting that the presence of the hydrogel suppressed the hydrophobic recovery of plasma-treated PDMS, but this method is tedious and time consuming. Chou et al. [16] grafted zwitterionic polymers by in situ self-assembling coating (ISC) which comprised polymerization and simultaneously coating of a copolymer onto the surface in order to resolve the solubility problem of zwitterionic copolymers. They applied ISC method to copolymers composed of hydrophobic styrene (ST) and hydrophilic sulfobetaine methacrylate (SBMA). They not only found that the coated surfaces resisted befouling but also improved wett ability. Nevertheless, this method required optimization of operating conditions including initial solid content in the polymerization bath, the monomer ratio and the reaction time.

In our lab group, 2-(trimethylsiloxy) ethyl methacrylate (TMSEMA) has been found to be a suitable reagent for hydrophilic surface medication of PDMS via hydrophilic reversal technique [17]. The reversal technique is a one-step solution soaking treatment that does not require any pretreatment or use of organic solvents. TMSEMA is mostly used as a protecting agent for hydroxyl groups in hydroxyethylmethacrylate due to the trimethylsiloxy groups contained in its structure. Mecerreyes et al. [18] fabricated the poly (alkyl methacrylate)-graft-polylactone via sequential living polymerization by utilization of TMSEMA protective effect on hydroxyl groups. Wang et al. [19] synthesized amphiphilic block copolymer PSt-b-PHEMA by hydrolysis of PSt-b-PTMSEMA that led to the synthesis of PSt-b-PHEMA metal hybrid. Liu et al. [20] prepared polystyrene-block-poly (2- hydroxyethyl methacrylate) with different molar masses via hydrolysis of trimethylsilyl groups and the micelles formed from these polymers in THF/cyclohexane mixtures were investigated. From the studies, it's clear that TMSEMA plays a critical role as a protecting agent for hydroxyl groups. We recently prepared series of PDMS film using (3-acryloxy-2-hydroxypropoxypropyl) terminated polydimethylsiloxane TMSEMA, then subjected the films to alkali hydrolysis to obtain hydrophilic PDMS surface with reduced protein adsorption [17].

The simplicity and convenience of TMSEMA hydrolysis reaction inspired us to apply TMSEMA to the hydrophilic surface modification of PDMS-PU for the first time. Unlike other previous PDMS modification methods, the proposed method renders hydrophilicity to PDMS-HEMA by surface reverse technique. The previously reported procedures have utilized organic solvents such as methanol, THF, cyclohexane etc. in efforts to hydrolyze TMSEMA [18-20], but the use of organic solvents results into contamination and increased toxicity which affects the materials if they are to be used in biological applications. Hence, an efficient and environmentally friendly method to hydrolyze TMSEMA is presented in this research.

In this paper, TMSEMA is key to hydrophilic surface modification of PDMS-HEMA films. A series of PDMS-PU films were fabricated using PDMS-HEMA macromer and a hydrophilizing reagent TMSEMA in the presence of a cross linker ethylene glycol dimethacrylate (EGDMA) and 2-Hydroxy-2- methylpiophenome (D-1173). The obtained PDMS-PU films were subjected to reversal technique using a certain wt. $\%$ of potassium hydroxide $(\mathrm{KOH})$ solution in order to obtain hydroxyl groups on the surface Figure 1.

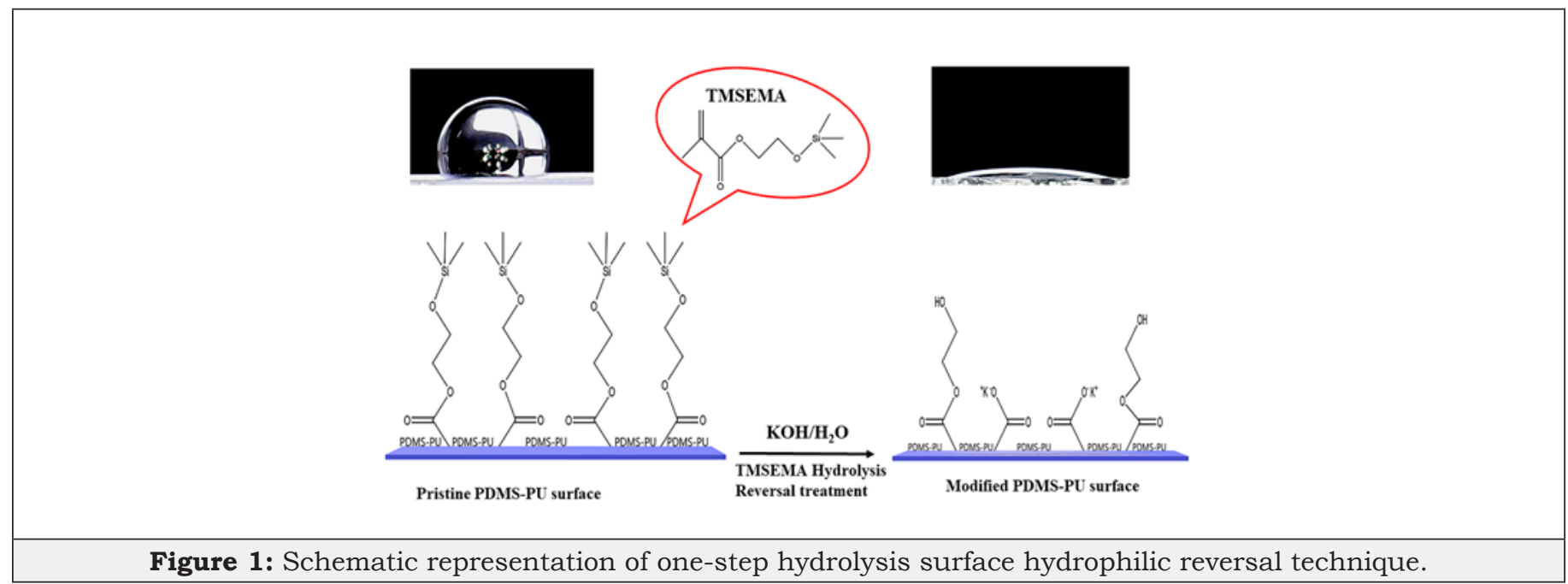




\section{Experimental Section}

\section{Materials}

Carbinol (hydroxyl) terminated polydimethylsiloxane (PDMS) was bought from Beijing HWRK Chem Co. Ltd, while 2-(trimethylsiloxy) ethyl methacrylate (TMSEMA) was bought from Alfa Aesar (China) Chemicals Co., Ltd. Isophoronediisocyanate (IPDI)was bought from Maya Reagent Co. Ltd, 2-hydroxyethyl methacrylate (HEMA) was obtained from Shanghai Dibo Chemical Technology Co. Ltd. 2-hydroxy-2-methylpiophenome (D-1173) and ethylene glycol dimethacrylate (EGDMA) were purchased from TCI (Shanghai) Development, potassium hydroxide $(\mathrm{KOH})$ was purchased from Shanghai Macklin Biochemical Industry Co. Ltd. Phosphate buffered saline (PBS) ( pH 7.4) was obtained from Sangon Biotech (Shanghai) Co. Ltd. Bicinchoninic acid (BCA) protein assay kit, bovine serum albumin (BSA)and lysozyme (LZM)were bought from Beyotime Biotechnology while sodium dodecyl sulfate (SDS) was bought from Shanghai Lingfeng Chemical Reagent Co. Ltd.

\section{Preparation of PDMS-HEMA macromer}

Carbinol (hydroxyl) terminated polydimethylsiloxane (PDMS) and the cross-linker isophoronediisocyanate (IPDI) in the ratio of 1:1.6 respectively were added into a flask, then a catalystdibutyltindilaurate (DBTDL) was added. The mixture was stirred for $4 \mathrm{~h}$ to allow the condensation reaction. 2-hydroxyethyl methacrylate (HEMA) in the ratio of 4:1 to IPDI and the initiator 2-hydroxy-2-methylpiophenome (D-1173) were introduced into the reaction mixture and the reaction mixture was further stirred for 2 $\mathrm{h}$ at room temperature in the dark.

\section{Preparation of the PDMS-PU films}

The PDMS-PU network was prepared by reacting the prepared PDMS-HEMA reaction mixture with different weight percentage ratios of TMSEMA using D-1173 and EGDMA as the photo-initiator and cross-linker respectively followed by $12 \mathrm{~h}$ stirring in the dark at room temperature $\left(25^{\circ} \mathrm{C}\right)$. Thereafter, the mixture was injected into the double stack polypropylene molds and UV-initiated $\left(\geq 15 \mathrm{mV} / \mathrm{cm}^{2}\right.$ ) for $2 \mathrm{~h}$ at room temperature. The resulting films were then stored in PBS buffer solution for further experiments. Pristine PDMS-PU containing TMSEMA was represented in Table 1.

Table 1: Copolymerization formulation for PDMS-PU film with different percentages (wt.\%) of hydrophilizing reagent TMSEMA.

\begin{tabular}{|c|c|c|c|c|c|}
\hline PDMS-PU & PPT-0 & PPT-1 & PPT-2 & PPT-3 & PPT-4 \\
\hline PDMS-HEMA & 97 & 93 & 89 & 85 & 81 \\
\hline TMSEMA & 0 & 4 & 8 & 12 & 16 \\
\hline EGDMA & 2.8 & 2.8 & 2.8 & 2.8 & 2.8 \\
\hline D-1173 & 0.2 & 0.2 & 0.2 & 0.2 & 0.2 \\
\hline
\end{tabular}

\section{PDMS-PU surface modification}

The PDMS-PU surface was modified via reversal treatment [17]. Under alkaline conditions, the trimethylsiloxy group contained in
TMSEMA undergoes hydrolysis to remove trimethylsilyl there by forming the hydroxyl group. The prepared films were treated using the optimum condition. Briefly, the prepared PDMS-PU films were immersed into $5 \mathrm{wt} \% \mathrm{KOH}$ solution and shaken at $150 \mathrm{rpm}$ for $3 \mathrm{~h}$ at $37^{\circ} \mathrm{C}$ to obtain the films. The films were washed with deionized water several times to remove the residues produced by the reversal treatment. Phosphate buffered saline was used for storing the obtained films for the next studies.

\section{Surface characterization of PDMS-PU films}

The water contact angle of the as-prepared PDMS-PU films was evaluated using a contact angle goniometer (KSV CAM-200, KSVIns), while attenuated total reflection Fourier transform infrared (Nicolet 5700 FTIR) spectroscopy was used to assess the functional groups difference on the surface of the unmodified and modified films. Furthermore, the surface morphology was examined using a scanning electron microscope (SEM FEI Inspect F50).

\section{Measurement of Equilibrium Water Content (EWC)}

The equilibrium water content of the PDMS-PU films was evaluated as follows:

$$
E W C(\%)=\frac{W_{w e t}-W_{d r y}}{W_{w e t}} \times 100
$$

Where $\mathrm{W}_{\text {wet }}$ is the weight of the wet sample and $\mathrm{W}_{d r y}$ is the weight of the dry sample. The sample was immersed in water for $24 \mathrm{~h}$ to obtain the weight of the wet sample.

\section{The mechanical properties}

A Labthink XLW (PC) Auto Tensile Tester was used to test the tensile strength and elongation at break of the films at a stretching rate of $50 \mathrm{~mm} / \mathrm{min}$.

\section{Protein adsorption analysis}

For protein adsorption analysis, the protein bovine serum albumin (BSA), lysozyme (LZM) solutions $(5 \mathrm{mg} / \mathrm{mL})$ and sodium dodecyl sulfate (SDS) solution (1wt. \%) prepared with pH 7.4 PBS buffer solutions and thereafter used to measure protein adsorption, with the static adsorption method being adopted. PDMS-PU films were immersed into protein solution $(3 \mathrm{~mL})$ and incubated at $37^{\circ} \mathrm{C}$ for $24 \mathrm{~h}$. The unabsorbed proteins were washed by transferring hydrogels into vials containing excess PBS buffer solution and shaken for 30 minutes at $30^{\circ} \mathrm{C}$. The hydrogels were intermittently washed thrice by changing the PBS buffer solution. Thereafter, the hydrogels were immersed in SDS solution ( $3 \mathrm{~mL}$ ) and shaken for $4 \mathrm{~h}$ at 37 ${ }^{\circ} \mathrm{C}$ to elude the adsorbed protein, after which the protein content was then, determined using the BSC protein kit by ELLIASA method.

\section{Results and Discussion}

In this research, we utilized the condensation reaction and ultraviolet initiated copolymerization reactions to prepare PDMS-PU films. Hydroxyl terminated PDMS was polymerized via condensa- 
tion reaction using IPDI as a cross-linker and DBTDL as a catalyst, HEMA was incorporated into the system by copolymerization using D-1173 as an initiator and further copolymerization of TMSEMA via ultraviolet copolymerization reaction using EGDMA as a crosslinker and D-1173 as a photo-initiator. A series of PDMS-PU films were fabricated using the formulation shown in Table 1. The as-prepared PDMS-PU films were hydrophilized by solution soaking in 5 wt. $\% \mathrm{NaOH}$ solution to allow reversal reaction. In this study, the maximum wt. \% content of TMSEMA used was $16 \mathrm{wt}$. \%, as it reached the desired hydrophilic effect.

\section{Water contact angle (WCA) analysis}

The effectiveness of the hydrophilic reversal reaction and the relationship between the content of TMSEMA and hydrophilic properties was studied by the WCA tests Figure 2. The WCA of PDMSPU without the TMSEMA content was $108^{\circ}$, while that of pristine PDMS-PU film with 16wt. \% TMSEMA was $103^{\circ}$. The WCA of the PDMS-PU film containing $4 \mathrm{wt}$. \% TMSEMA decreased significantly upon the surface reversal treatment. Further, increasing TMSEMA content resulted in further reduction in the WCA with $16 \mathrm{wt}$. \% content reducing the WCA to $16^{\circ}$, which is a more hydrophilic state. Meanwhile, the WCA of the PDMS-PU film without TMSEMA did not change after the same treatment. From this result, it can be concluded that the hydrophilizing reagent TMSEMA has no hydrophilic modification effect on the non-reversal-treated PDMS-PU, which could be attributed to the siloxane structure in TMSEMA making it hydrophobic and couldn't affect the wetting effect of the PDMS-PU. From Figure 2B, MPT-3 and MPT-4 has better hydrophilicity hence selected for studying the hydrophobic recovery of the modified PDMS-PU. A slight but insignificant increase of WCA of bothMPT-3 and MPT- 4 was observed after 60 days of storing the films in PBS solution compared to the first day of treatment, indicating that the hydroxyl groups generated by the reversal reaction remained on the surface, thereby stabilizing the hydrophilicity of the PDMSPU films. Therefore, the proposed surface reversal treatment is an efficient and feasible method as it achieved a stable and excellent hydrophilic effect.
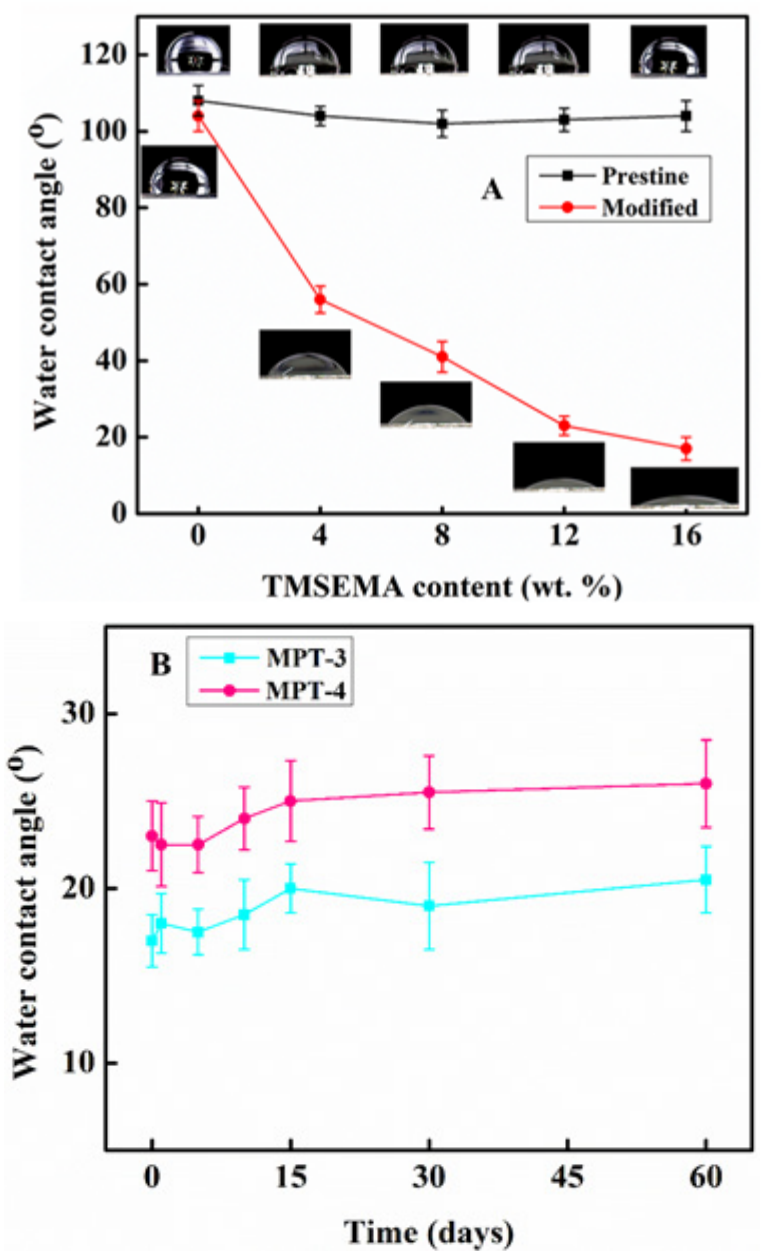

Figure 2: Static water contact angles: A, pristine PDMS-PU and modified PDMS-PU films as the function of wt. \% TMSEMA; B, hydrophobic recovery of the modified PDMS-PU films (MPT-3 and MPT-4) as the function of time.

\section{ATR-FTIR surface analysis}

The contact angle measurement confirmed that, after the hy- drophilic reversal treatment, the hydrophilicity of the PDMS-PU films improved significantly and this was attributed to the genera- 
tion of the hydroxyl groups on the surface upon reversal treatment. Therefore, to prove this, the surface of the PDMS-PU film was tested by ATR-FTIR to monitor the generation of the hydroxyl groups on the surface. From Figure 3 it can be seen that for both pristine and modified PDMS-PU, the absorption bands at $\left(\sim 798 \mathrm{~cm}^{-1}\right)$ and $\left(\sim 1261 \mathrm{~cm}^{-1}\right)$ are ascribed to $\mathrm{SiCH}_{3}$ stretching vibrations and bending deformation respectively, while $\left(\sim 1022 \mathrm{~cm}^{-1}\right)$ and $\left(\sim 1091 \mathrm{~cm}^{-1}\right)$ are attributed to symmetric and asymmetric -Si-O-Si- stretches, respectively. The absorption peak appearing at $\left(\sim 1727 \mathrm{~cm}^{-1}\right)$ is due to the $-\mathrm{C}=0$ stretching vibration. The modified PDMS-PU had the absorption appearing at $\left(\sim 3387 \mathrm{~cm}^{-1}\right)$ which is ascribed to the $-\mathrm{OH}$, the peak intensity increased with increased content of TMSEMA. The principle of the hydrophilic reversal treatment method and its hydrophilic effect is supported by the presence of the hydroxyl groups. Furthermore, the absorption peak at $\left(\sim 1568 \mathrm{~cm}^{-1}\right)$ was observed and its intensity increased with increasing TMSEMA content. This observation could be ascribed to the reversal reaction not only causing the cleavage of the $\mathrm{Si}-\mathrm{O}$ bond to form the hydroxyl group, but also resulted in the formation of the ester bond due the hydrolysis of TMSEMA in the alkaline solution to form salt (carboxyl ate) structure.

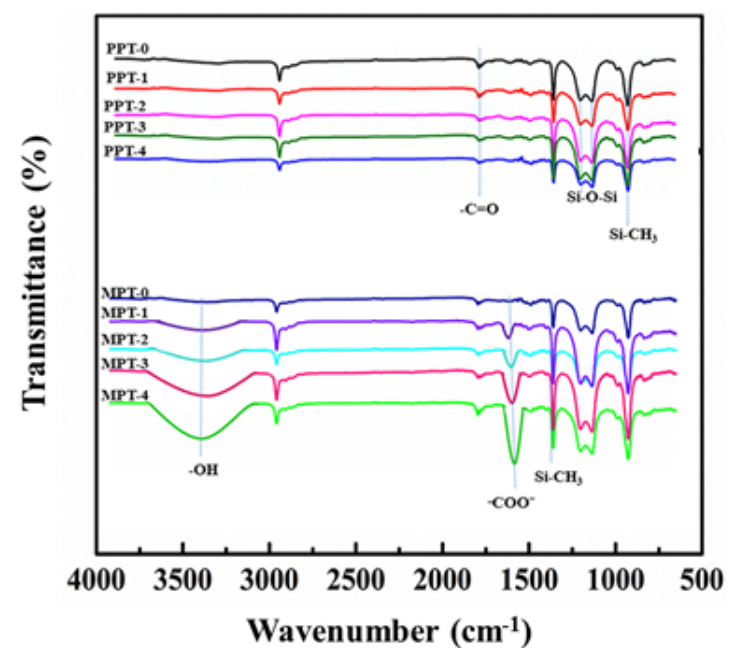

Figure 3: ATR-FTIR spectra of PDMS-PU films surface with different wt. \% TMSEMA of contents: pristine PDMS-PU (PPT-0 to PPT-4); modified PDMS-PU (MPT-0 to MPT-4).

\section{Equilibrium water content (EWC) analysis}

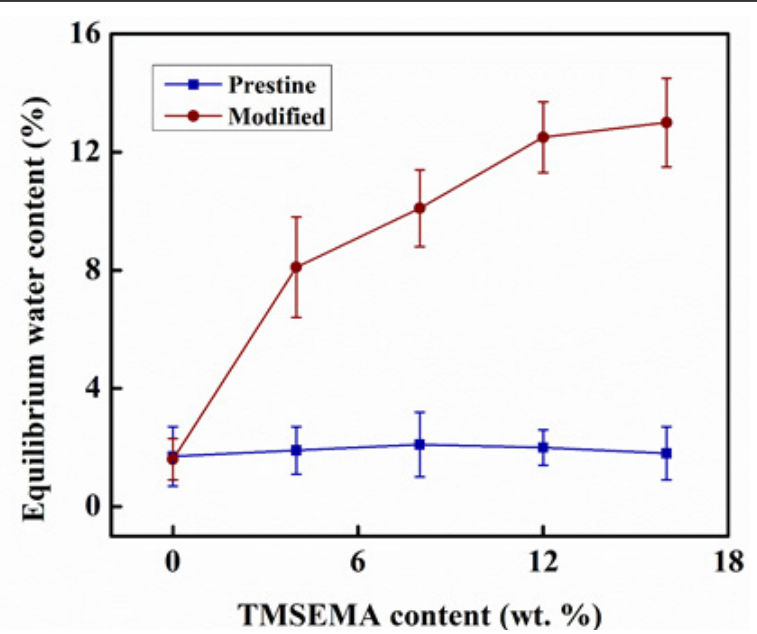

Figure 4: Equilibrium water content of PDMS-PU films as the function of wt. \% TMSEMA content for both pristine PDMS-PU and modified PDMS-PU.

The EWC test was performed in order to study the effect of the hydrophilizing reagent and the surface reversal treatment on the swelling behavior of the PDMS-PU films Figure 4. The EWC of the pristine PDMS-PU film without TMSEMA content was $1.7 \%$ and the EWC of the pristine film did not increase with increasing TMSEMA content, as it remained almost constant at around the same value.
However, the EWC value of the modified PDMS-PU films increased gradually with increasing content of TMSEMA to $13 \%$ at $16 \mathrm{wt}$. \% TMSEMA content. The EWC results are similar to that of the WCA in which the hydrophilic reversal treatment had no obvious effect on the PDMS-PU film without TMSEMA. It was also observed that, the change in the EWC was not as significant in comparison to the pris- 
tine PDMS-PU. Although the EWC of the modified films increased, the increase still remained at low limits in comparison to the sharp decrease observed in the WCA, and this can be attributed to the hydrophobic nature of the PDMS. Therefore, the proposed treatment method only caused the generation of the hydrophilic hydroxyl groups on the surface of the film, while the interior structure of the PDMS-PU was still based on the naturally hydrophobic siloxane structure which makes it difficult for the water to penetrate the interior of the film after immersion in water, thereby leading to an insignificant rise in the EWC.

\section{Surface morphology}

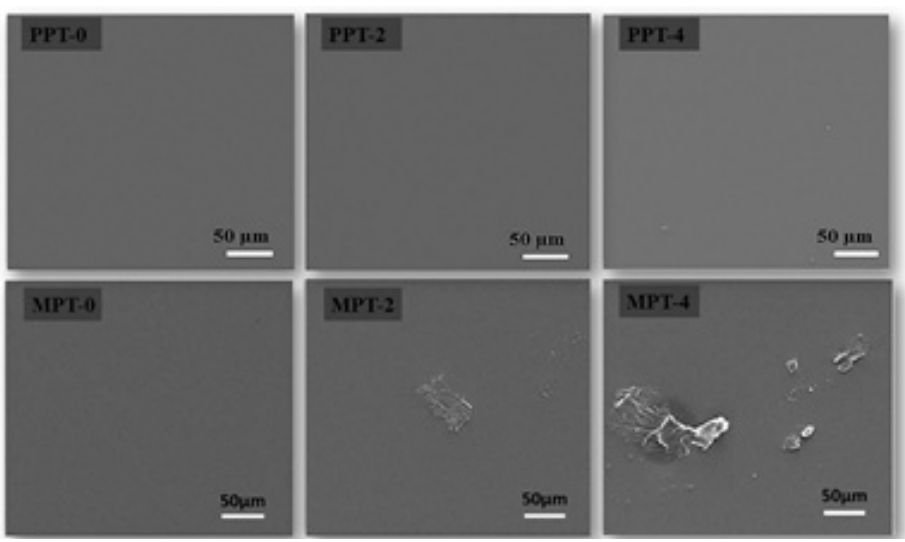

Figure 5: SEM images of PDMS-PU films with different TMSEMA contents: pristine PDMS-PU (PPT-0, PPT-2 and PPT4) and modified PDMS-PU (MPT-0, MPT-2 and MPT-4).

The pristine and modified PDMS-PU film's surface morphologies were studied by SEM analysis. Figure 5 depicts the surface morphologies of the PDMS-PU before and after reversal treatment. As can be seen from the SEM images, a smooth and flat surface was observed for pristine PDMS-PU and the increase in the TMSEMA content had no observable effect on the film surface. However, after the reversal treatment, a slightly rough surface was observable for the modified PDMS-PU surface and the increase in TMSEMA had a corresponding increase in the damage or defects on the film surface, though most of the surface remained flat. Therefore, the $\mathrm{KOH}$ solution soaking treatment caused some minor but significant defects on the surface of the modified PDMS-PU films.

\section{Mechanical properties}

To study the effect of the $\mathrm{KOH}$ solution treatment on the mechanical properties of the modified PDMS-PU films, the tensile strength and elongation at break of both the pristine and modified PDMS-PU films were determined. As can it be seen from Figure $6 \mathrm{~A}$ $\& 6 \mathrm{~B}$, both the tensile strength and the elongation at break of the pristine PDMS-PU films increased with increasing TMSEMA content. This could be attributed to the compatibility of TMSEMA with the PDMS-PU as supported by the smoothness and flatness of the pristine SEM images. The copolymerization of the TMSEMA to the PDMS-HEMA system could have increased the cross-linking density which is a contributing factor to the increased tensile strength and elongation at break. The modified PDMS-PU films showed a general increasing trend in tensile strength with increasing TMSEMA content, however, compared to the pristine PDMS-PU, the modified PDMS-PU tensile strength decreased for every point of increase in TMSEMA content Fig. 6A. The elongation at break for the modified
PDMS-PU film showed a decreasing trend with increasing content of TMSEMA Figure 6B. The observed trend can be ascribed to the defects observed on the surface of the modified PDMS-PU caused by $\mathrm{KOH}$ solution treatment. Other researchers have reported similar results when they studied mechanical properties [21-23].

\section{Evaluation of protein adsorption}

Static adsorption method was carried out in this study; BSA and LZM were the selected model proteins. In order to eliminate the influence of external factors on different experiments, the PDM-PU film without TMSEMA content (PPT-0) was chosen as the control surface and its adsorption intensity regarded as $100 \%$. The relative protein adsorption ratios pristine and modified PDMS-PU films are shown in Figure 6. From Figure 7A, it can be seen that the relative ratios of the BSA adsorption of pristine PDMS-PU films containing different wt. \% TMSEMA content fluctuated at $100 \%$. This indicates that, there was no change in the surface groups of the PDMS-PU films before and after addition of TMSEMA, as can be seen from the ATR-FTIR spectrum, which is also consistent with siloxane structure contained in TMSEMA. Nevertheless, the relative ratio of BSA adsorption of the modified PDMS-PU films decreased significantly with increasing content of TMSEMA. The relative ratio of BSA adsorption decreased to $14 \%$ when the TMSEMA content reached the maximum value of $16 \mathrm{wt}$. \%. However, in contrast with the observed trend of the BSA relative adsorption ratio, the relative ratio of LZM adsorption of PDMS-PU films with different content of TMSEMA did not change while increasing trend in the relative ratio of LZM adsorption of the modified PDMS-PU films was observed in Figure 7B. According to the isoelectric point theory (IP), the isoelectric points of LZM and BSA are 11.0 to 13.5 and 4.7 respectively, therefore, in 
PBS solution pH 7.4, LZM is positively charged while BSA is negatively charged [18,24]. From the IR spectrum in Figure 3, the carboxyl ate and hydroxyl functional groups are generated on the surface of the PDMS-PU after the hydrophilic reversal treatment, which results in the modified PDMS-PU surface being negatively charged. Further, the IR spectrum shows that the intensity of the carboxyl ate groups on the surface of the surface of the reversal-treated
PDMS-PU is significantly enhanced with increasing TMSEMA content, which explains why the relative ratio of BSA adsorption decrease with TMSEMA content, while that of LZM increases. These results are in agreement with those reported by other researchers who studied protein adsorption [18,24-26]. Many researchers have reported that, hydrophilic surfaces are more resistant to protein adsorption compared to hydrophobic surfaces [25,27-29].
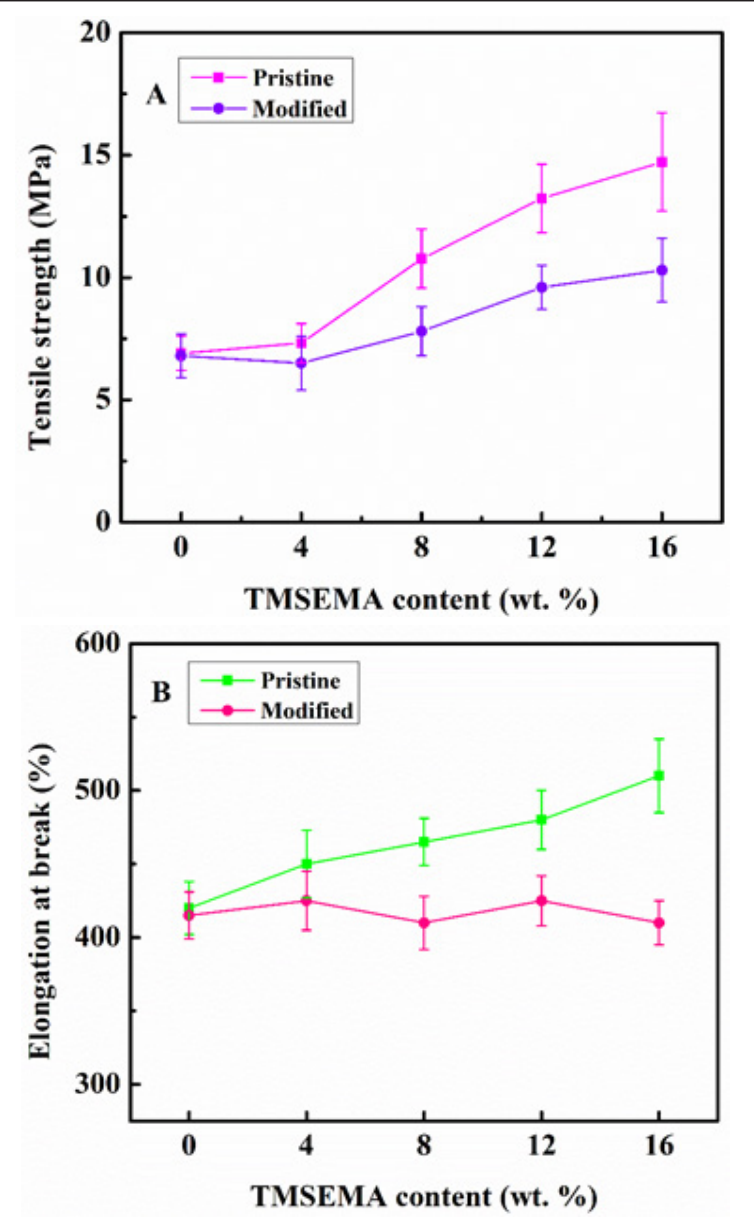

Figure 6: Mechanical properties of PDMS-PU films (A). Tensile strength of the pristine and modified PDMS-PU at different TMSEMA content (wt. \%) (B). Elongation at break of the PDMS-PU at different TMSEMA content (wt. \%).

However, in the current study, the protein adsorption capacity of the reversal-treated PDMS-PU surface was not only dependent on its hydrophilicity but also on the protein isoelectric point value resulting from the surfaces negatively charged characteristics. Consequently, the lipophilicity of the hydrophilizing reagent TMSEMA provides it with good compatibility with the hydrophobic siloxane structure, and can be added in any proportion in the synthesis of the PDMS-PU film system. TMSEMA's low surface energy properties enables it to migrate to the surface and accumulate on the surface of the film to allow the reverse reaction to take place. Further, the generation of the carboxyl and hydroxyl functional groups on the modified film surface offers more potential and possibility for further modification of PDMS-PU.

\section{Conclusion}

A series of PDMS-PU films were prepared via the copolymerization of hydrophilizing reagent TMSEMA and the macromer PDMS-HEMA. The as-prepared PDMS-PU films were subjected to one-step reversal treatment. The WCA measurements on the PDMS-PU surface indicated that the wet ability was greatly improved after the reversal treatment. The improvement of the wettability was further confirmed by ATR-FTIR analysis, which showed the formation of the hydroxyl and carboxyl ate groups on the reversal-treated PDMS-PU surface. Meanwhile, the EWC analysis showed that the water holding capacity of the PDMS-PU did not significantly increase after modification, indicating that the hydrophilic reverse reaction only hydrolyzes the surface while the interior of the PDMS-PU remains the hydrophobic siloxane structure. Further, the 
SEM analysis showed that the alkaline solution soaking treatment caused damage on the sample surface. The damage caused on the surface slightly decreased both the tensile strength and the elongation at break of the PDMS-PU films. The modified PDMS-PU showed different protein adsorption performance towards different protein. The relative ratio of BSA adsorption decreased with increas- ing TMSEMA while the relative ratio of LZM adsorption exhibited a general upward trend. This study explored a non-polluting, simple, efficient and cost effective method for improving the hydrophilicity of PDMS-PU for biomaterial applications. It is hoped that this method will in the near future be applied to hydrophilic surface modification of other PDMS materials.

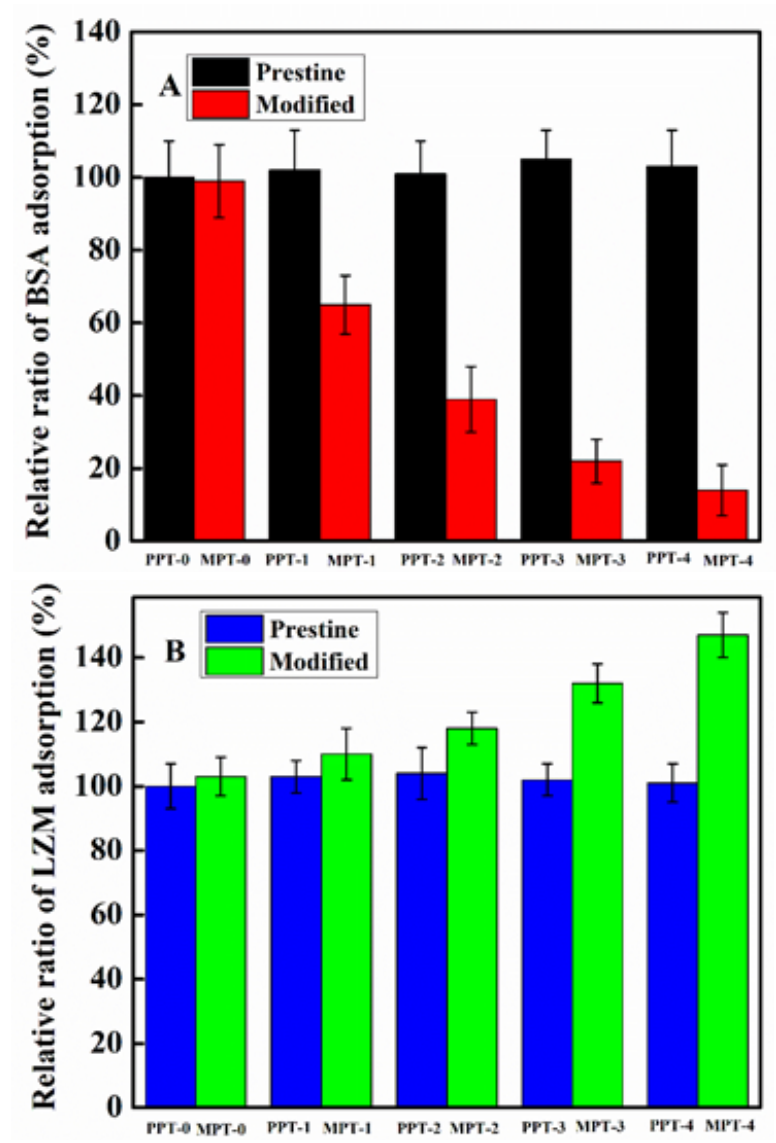

Figure 7: Relative ratio of protein adsorption of PDMS-PU films with different wt. \% TMSEMA content: A, BSA adsorption and B, LZM adsorption.

\section{Acknowledgement}

This work was supported by the National Natural Science Foundation of China (NSFC) with grant number 21174029, the Industry Academia Cooperation Innovation Fund of Jiangsu Province with grant number BY2014127-07, the Project Funded by the Priority Academic Program Development of Jiangsu Higher Education Institutions (PAPD), and the Fundamental Research Funds for the Central Universities with grant number 2242016K41020. Special gratitude goes to The University of Zambia, Chemistry Department for the enabling environment and support rendered towards this research.

\section{References}

1. Trantidou T, Elani Y, Parsons E, Ces O (2017) Hydrophilic surface modification of PDMS for droplet micro fluidics using a simple, quick, and robust method via PVA deposition. Microsystems \& Nanoengineering 3: 16091.

2. Xue P (2018) A simple technique of constructing nano-roughened polydimethylsiloxane surface to enhance mesenchymal stem cell adhesion and proliferation. Micro fluidics and Nanofluidics 22.
3. Maruko Y, Guadalupe PGT, Tagaya M (2018) Modification of poly(dimethylsiloxane) by mesostructured siliceous films for constructing protein-interactive surfaces. E-Journal of Surface Science and Nanotechnology 16: 41-48.

4. Kalulu M, Zhang W, Xia XK, Oderinde O, Jiang Y (2018) Hydrophilic surface modification of polydimetylsiloxane-co-2-hydroxyethylmethacrylate (PDMS-HEMA) by Silwet L-77 (heptamethyltrisiloxane) surface treatment. Polymers for Advanced Technologies.

5. Lee D, Yang S (2012) Surface modification of PDMS by atmospheric-pressure plasma-enhanced chemical vapor deposition and analysis of long-lasting surface hydrophilicity. Sensors and Actuators B: Chemical 162: $425-434$

6. Juárez-Moreno JA, Ávila-Ortega A, Oliva AI, Avilés F, Cauich-Rodríguez JV (2015) Effect of wettability and surface roughness on the adhesion properties of collagen on PDMS films treated by capacitively coupled oxygen plasma. Applied Surface Science 349: 763-773.

7. Seo KS (2015) Surface treatment of glass and poly (dimethylsiloxane) using atmospheric-pressure plasma jet and analysis of discharge characteristics. Japanese Journal of Applied Physics 54: $01 \mathrm{AE} 06$.

8. Tian K, Bae J, Suo Z, Vlassak JJ (2018) Adhesion between hydrophobic elastomer and hydrogel through hydrophilic modification and interfacial segregation. ACS Applied Materials \& Interfaces 10: 43252-43261. 
9. Sanli D, Erkey C (2013) Monolithic composites of silica aerogels by reactive supercritical deposition of hydroxy-terminated poly (dimethylsiloxane). ACS Applied Materials and Interfaces 5: 11708-11717.

10. Eddington DT, Puccinelli JP, Beebe DJ (2006) Thermal aging and reduced hydrophobic recovery of polydimethylsiloxane. Sensors and Actuators B: Chemical 114: 170-172.

11. Qin M (2007) Bioactive surface modification of mica and poly (dimethylsiloxane) with hydrophobins for protein immobilization. Langmuir 23 4465-4471.

12. Sui G (2006) Solution-phase surface modification in intact poly (dimethylsiloxane ) microfluidic channels. 78: 5543-5551.

13. Bodas D, Khan-Malek C (2007) Hydrophilization and hydrophobic recovery of PDMS by oxygen plasma and chemical treatment-An SEM investigation. Sensors and Actuators B: Chemical 123: 368-373.

14. Pinto S (2010) Poly (dimethyl siloxane) surface modification by low pressure plasma to improve its characteristics towards biomedical applications. Colloids and Surfaces B: Biointerfaces 81: 20-26.

15. Lin $\mathrm{CH}$, Cho HL, Yeh YH, Yang MC (2015) Improvement of the surface wet ability of silicone hydrogel contact lenses via layer-by-layer self-assembly technique. Colloids and surfaces B Biointerfaces 136: 735-743.

16. Chou YN (2018) Surface zwitterionization on versatile hydrophobic interfaces via a combined copolymerization/self-assembling process Journal of Materials Chemistry B 6: 4909-4919.

17. Zhang W (2018) Reverse hydrophobic PDMS surface to hydrophilic by 1-step hydrolysis reaction. Polymers for Advanced Technologies 29: 2103-2109.

18. Mecerreyes D, Dubois P, Jérôme R, Hedrick JL (1999) Synthesis of well-defined poly (alkyl methacrylate)-graft-polylactone by sequential living polymerization. Macromolecular Chemistry and Physics 200: 156165.

19. Wang Y, Wu J, Yuan J, Sun G, Pan C (2002) Preparation and characterization of PSt-b-PHEMA metal hybrids. Journal of Applied Polymer Science 83: 2883-2891.
20. Liu G, Smith CK, Hu N, Tao J (1996) Formation and properties of polystyrene- block -poly (2-hydroxyethyl methacrylate) micelles. Macromolecules 29: 220-227.

21. Wala J, Maji D, Das S (2017) Influence of physico-mechanical properties of elastomeric material for different cell growth. Biomedical Materials (Bristol) 12.

22. Soulas DN, Sanopoulou M, Papadokostaki KG (2013) Hydrophilic modification of silicone elastomer films: Thermal, mechanical and theophylline permeability properties. Materials Science and Engineering C 33: 2122-2130.

23. Zhang X, Lin G, Kumar SR, Mark JE (2009) Hydrogels prepared from polysiloxane chains by end linking them with tri functional silanes containing hydrophilic groups. Polymer 50: 5414-5421.

24. Xie H, Zhao Z, An S, Jiang Y (2015) The influence of the surface properties of silicon-fluorine hydrogel on protein adsorption. Colloids and Surfaces B: Biointerfaces 136: 1113-1119.

25. Perry CC, Roach P, Farrar D (2006) Surface tailoring for controlled protein adsorption: Effect of topography at the nanometer scale and chemistry. Journal of the American Chemical Society 128: 3939-3945.

26. Zhao Z (2013) Effect of surface compositional heterogeneities and micro phase segregation of fluorinated amphiphilic copolymers on antifouling performance. ACS Applied Materials and Interfaces 5: 7808-7818.

27. Chen S, Li L, Zhao C, Zheng J (2010) Surface hydration: Principles and applications toward low-fouling/nonfouling biomaterials. Polymer 51: 5283-5293.

28. Wu HX, Zhang XH, Huang L, Ma LF, Liu CJ (2018) Diblock polymer brush (pheaa-b-pfma): microphase separation behavior and anti-protein adsorption performance. Langmuir 34: 11101-11109.

29. Fuchiwaki Y (2015) Batch quartz crystal microbalance immunosensor using a protein-immobilized hydrophilic polymer. Analytical Letters 48: 1570-1577. 